\title{
Optical Properties of SOI Waveguides Functionalized with Close-Packed Quantum Dot Films
}

\author{
Zeger Hens, Abdoulghafar Omari, Pieter Geiregat, Dries Van Thourhout \\ Physics and Chemistry of Nanostructures, Ghent University, Belgium \\ Center for Nano and Biophotonics, Ghent University, Belgium \\ Photonics Research Group, Ghent University, Belgium \\ Tel: (32) 92644863,Fax: (32) 92644983,e-mail: Zeger.Hens@UGent.be
}

\begin{abstract}
It is shown that dipolar coupling between neighboring quantum dots enhances the absorption of light in close packed monolayers of colloidal quantum dots. Based on this concept, the experimentally determined losses in planarized waveguides coated by a quantum dot monolayer can be successfully simulated. These simulations rely on replacing the quantum dot layer by an effective medium with a dielectric function determined by dipolar coupling and use the dielectric constant of the quantum dot host medium as the only adjustable parameter. This leads to a generic approach for the simulation of optical materials including close packed quantum dot layers.
\end{abstract}

Keywords: functionalized waveguides, colloidal quantum dots, optical losses, silicon, telecommunication.

\section{INTRODUCTION}

Colloidal quantum dots offer a unique combination of size-tunable optical properties and a suitability for solution-based processing [1]. This implies that their properties can be fit to the application and that they can be readily combined with a variety of materials or technology platforms. These include silicon-on-insulator (SOI) and silicon nitride based integrated photonic circuits, where colloidal quantum dots can be used for the on-chip generation, detection or processing of light. These applications typically rely on close-packed mono- or multilayers of quantum dots.

In this paper, we analyse the optical properties of such quantum dot layers. We first start from absorption measurements, where we find that the absorption cross section of a quantum dot in a close packed film can be enhanced by a factor of 5 relative to the value found in a dilute dispersion. We show that an effective medium model that explicitly includes the dipolar coupling between neighboring quantum dots can account for this enhancement and its particular dependence on the quantum dot size and the quantum dot material. In a second step, we use this effective medium model to simulate experimentally determined losses in planarized SOI waveguides coated by monolayers of quantum dots. Using the host dielectric constant as the only adjustable parameter, we find excellent correspondence between the experimental losses and the simulation results for dielectric constants of the host in the range $1-2$, a reasonable value for quantum dots coated by apolar organic ligands. As such, this work provides the conceptual basis needed for the optical simulation of hybrid photonic devices based on thin films of colloidal quantum dots.

\section{EXPERIMENTAL}

The oleate-capped $\mathrm{PbS}$ and $\mathrm{PbS} / \mathrm{CdS}$ quantum dots used in this work were synthesized using established literature procedures [2],[3] and subsequently deposited as close packed monolayers using Langmuir-Blodgett deposition [4]. Importantly, this leads to the formation of large area, homogeneous films both on glass substrates (see Fig. 1A) and on silicon-on-insulator chips, where local deposition is achieved in combination with optical lithography[5].

When deposited on glass substrates, the absorption cross section $\sigma_{f}$ of a quantum dot can be determined from the film absorbance $A$, corrected for the film reflectance $R$ :

$$
\sigma_{f}=\ln 10 \times \frac{A-R}{N_{s}} .
$$

Here, $N_{\mathrm{s}}$ denotes the surface density of quantum dots, a number that can readily be determined using transmission electron microscopy (TEM). In equation (1), scattering is neglected since the wavelengths used $(>400 \mathrm{~nm})$ are much larger than the quantum dot diameter. The correction of the absorbance for reflection is typically very small $(<10 \%)$.

When deposited on waveguides, the net absorption coefficient $\alpha$ of the QDs is obtained by loss measurements on waveguides covered by a strip of QDs with varying length $L$ (see Fig. 2B). Using one of the waveguides as a reference, the transmitted power $P_{t}$ in the other waveguides can be expressed as:

$$
\log \frac{P_{t, r e f}}{P_{t}}=\alpha\left(L-L_{r e f}\right) .
$$


A

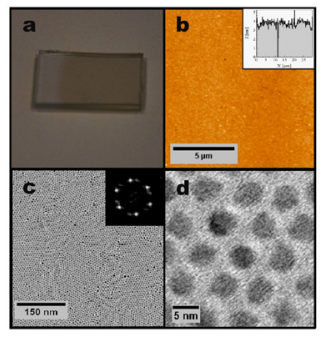

B

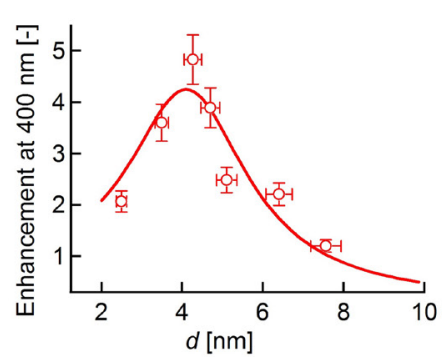

C

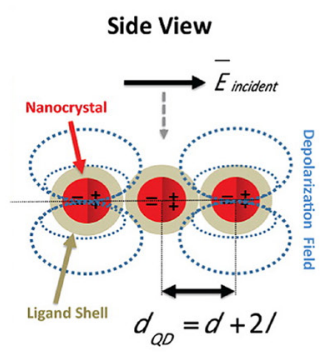

D

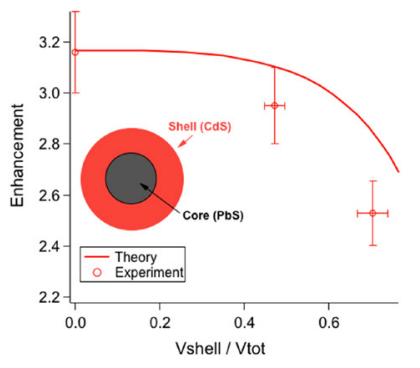

Figure 1: (A) Langmuir-Blodgett monolayer of $\mathrm{PbS}$ quantum dots, including (a) a contrast picture of a monolayer of $\mathrm{PbS}$ quantum dots $(d=5 \mathrm{~nm})$ on a glass surface $(2 \times 1 \mathrm{~cm})$ showing homogeneous $\mathrm{cm}^{2}$ coverage; (b) an atomic force microscope scan of the same PbS monolayer indicating excellent area uniformity (inset: cross section); (c) a larger area TEM image showing the PbS quantum dot superlattice with local hexagonal ordering (inset: Fourier transform image) and (d) a zoomedin TEM image showing the individual quantum dots and their interdistance. (B) Absorption enhancement for PbS quantum dots stabilized by oleate ligands at $400 \mathrm{~nm}$ as a function of QD diameter $d$. The full line represents the predictions based on the coupled dipole model. (C) Side view schematic of monolayer subject to incident field polarized in-plane. The relevant geometrical parameters are indicated by $d_{Q D}, d$, and $l$ as the (nearest-neighbor) interdot distance, QD size, and ligand length, respectively. Absorption enhancement for $\mathrm{PbS} / \mathrm{CdS}$ core/shell quantum dots as a function of the ratio between shell volume and total volume. The full line represents the enhancement according to the coupled dipole model.

Following equation (2), we calculate $\alpha$ from the slope of a $\log P_{t, r e f} / P_{r e f} v s . L-L_{r e f}$ plot.

\section{RESULTS}

In Figure 1B, we plot the ratio between $\sigma_{f}$ measured on monolayers of PbS QDs at $400 \mathrm{~nm}$ and the absorption cross section $\sigma_{0}$ of the same quantum dots in a dilute dispersion in tetrachloroethylene [6]. We find that this ratio, which we call the enhancement $E$, shows a marked dependence on the QD diameter, with a maximum value of about 5 for $4 \mathrm{~nm}$ PbS QDs. As shown before, this enhanced absorption of quantum dots in monolayers and its size dependence finds its origin in the dipolar coupling of the polarization fields in neighbouring quantum dots induced by the incident optical field (see Fig. 1C) [7]. This results in the following expression for $\sigma_{f}$ :

$$
\sigma_{f}=\frac{2 \pi}{\lambda n_{h}} \operatorname{Im}\left(\frac{a_{0, h}}{1-\frac{a_{0, h}}{\varepsilon_{h}} S}\right) .
$$

Here, $\lambda$ is the wavelength of light, $n_{h}$ and $\varepsilon_{h}$ are the refractive index and the dielectric constant of the host in which the QDs are embedded, respectively while $a_{0, h}$ is the polarizability of an isolated quantum dot in the host medium and $S$ is the so-called dipole sum, a term grouping the dipolar contributions of the neighboring particles to the field that drives a given QD. As shown by the full line in Fig. 1B, equation (3) reproduces the experimentally determined absorption enhancement when taking $\varepsilon_{h}=1.5$ and a QD size dispersion of $10 \%$. This correspondence indicates that the optical properties of QDs in close packed arrays indeed strongly depend on dipolar coupling between neighboring QDs. Importantly, the same expression also accounts for the absorption enhancement measured with $\mathrm{PbS} / \mathrm{CdS}$ core/shell QDs in close-packed monolayers (see Fig. 1D), provided that the appropriate expression for the polarizability of a core/shell nanocrystals is used.

In Fig. 2, we show the results of loss measurements in the wavelength range $1.48-1.56 \mu \mathrm{m}$ of planarized SOI waveguides coated with a close packed monolayer of $\mathrm{PbS} / \mathrm{CdS}$ QDs. For these experiments, core/shell QDs were chosen with a maximum of their first exciton absorbance at $1450 \mathrm{~nm}$ (see Fig. 2A). In this way, we will probe the well discernible long wavelength tail of these QDs. By measuring waveguides coated with QD strips of different length (Fig. 2B), we obtain the waveguide absorbance related to the presence of the QDs as plotted in Fig. 2C. We find, depending on the wavelength, absorption coefficients in the range $2-5 \mathrm{~cm}^{-1}$, that clearly follow the slope of the first exciton absorbance of the QDs in this wavelength range.

To compare the experimental QD with model predictions, we use an approach where the real QD layer covering the PWG is replaced by an effective medium with a dielectric function $\varepsilon_{e f f}$ (see Fig. 3A). Using the real geometry of the PWG - which includes a slightly submerged waveguide top surface, coated by a thin native silica layer - this enables us to extract a theoretical absorption coefficient $\alpha$ from the simulated effective refractive index $\tilde{n}_{\text {eff }}=n_{\text {eff }}+i \kappa_{\text {eff }}$ of the propagating quasi-TE mode:

$$
\alpha=\frac{4 \pi \kappa_{e f f}}{\lambda} .
$$


A

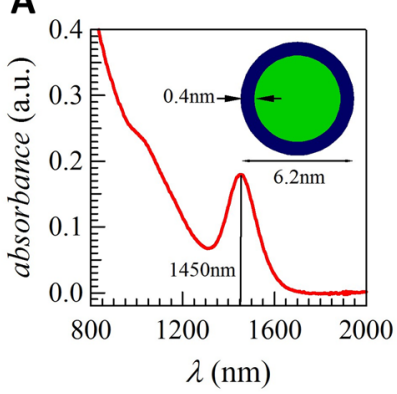

B

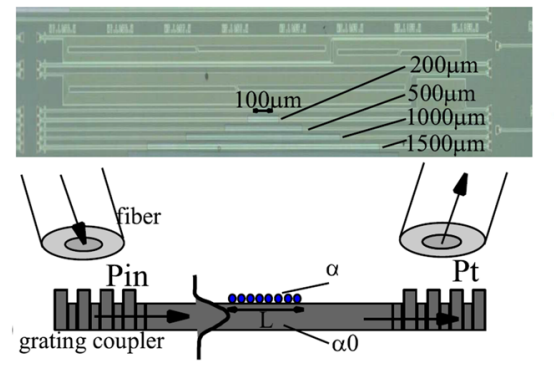

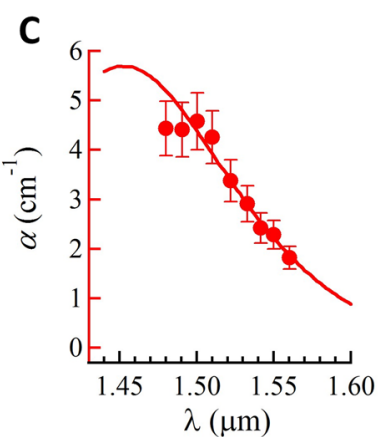

Figure 2: (A) Absorption spectrum of the $\mathrm{PbS} / \mathrm{CdS}$ QD used here, as recorded on a dilute QD dispersion in tetrachloroethylene. (B) Optical microscopy image of a sample with planarized waveguides coated by a QD monolayer with various strip lengths and a cartoon representation of the optical field coupled from the fiber through the grating in the QD coated PWG. (C) Absorption coefficient of the QD coated waveguides related to the presence of the QDs. The full line represents the QD absorption spectrum, scaled to the measured waveguide absorption coefficient.

This approach however requires that the dielectric function of each material or medium involved is known. For silicon and silica, we use typical values at $1520 \mathrm{~nm}$ of 3.45 and 1.45 , respectively. For $\varepsilon_{e f f}$, we use the expression based on the coupled dipole modelled introduced above:

$$
\varepsilon_{e f f}=\varepsilon_{h}\left(\varepsilon_{0}+\frac{N_{s}}{\delta} \frac{a_{0, h}}{\varepsilon_{h}-a_{0, h} S}\right) .
$$

Here, $\delta$ denotes the assumed thickness of the effective layer. Importantly, the dipole sum $S$ is in general different for fields parallel $\left(S_{\|}\right)$or perpendicular $\left(S_{\perp}\right)$ to the QD film. However, since the main field component of the quasi-TE modes in the PWG used here lies parallel to the QD film, only $S_{\|}$- which was also used for the analysis of the absorption enhancement (see Fig. 1) is of relevance here.

Opposite from $S$, which only depends on the position of the particles relative to each other, $a_{0, h}$ is a function of $\varepsilon_{h}$ and the dielectric function $\varepsilon_{Q D}=\varepsilon_{Q D, \mathrm{Re}}+i \varepsilon_{Q D, \mathrm{Im}}$ of the QDs. While we consider $\varepsilon_{h}$ as an adjustable parameter in this study, we use calculated values for $\varepsilon_{Q D, \mathrm{Re}}$ and $\varepsilon_{Q D, \mathrm{Im}}$, taking care that they yield the experimental absorption coefficient spectrum of the QDs in a dilute dispersion while obeying the KramersKronig transformation [8]. Importantly, in this analysis, we assume that the absorption coefficient of the $\mathrm{PbS} / \mathrm{CdS}$ core/shell QDs at wavelengths shorter than $400 \mathrm{~nm}$ can be derived from the bulk dielectric function of $\mathrm{PbS}$ and $\mathrm{CdS}$, respectively - as was demonstrated for $\mathrm{PbSe} / \mathrm{CdSe}$ QDs [9] - and we neglect possible quantization effects in the CdS shell.

Combining the geometry of the PWG cross section and the expression for $\varepsilon_{\text {eff }}$ - based on the coupled dipole model and the self-consistently determined $\varepsilon_{Q D}$ - the electric field of the guided optical mode in the PWG can be calculated, resulting in theoretical values for $\widetilde{n}_{\text {eff }}$ and $\alpha$. As an example, Fig. $3 \mathrm{~B}$ represents the electric field at a wavelength of $1520 \mathrm{~nm}$ for a PWG covered by a QD monolayer as obtained using Fimmwave 3.4 complex mode solver. The figure clearly shows the overlap between the QD film and the evanescent field, which makes that light absorption by the QDs affects $\kappa_{\text {eff }}$ and leads to a non-zero $\alpha$.

A

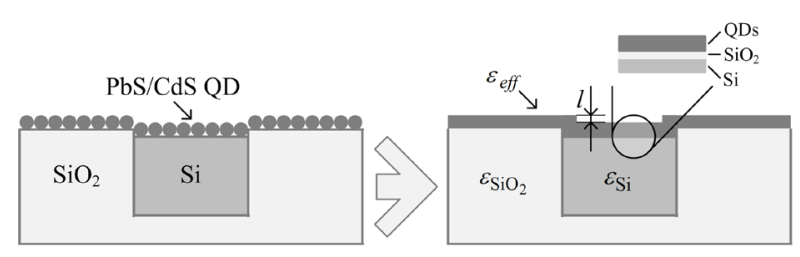

B

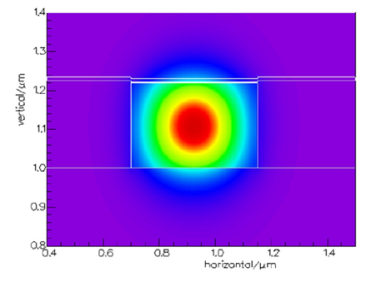

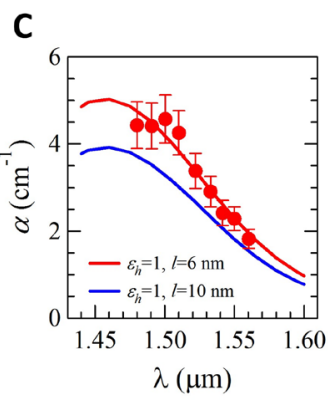

Figure 3: (A) Cartoon representation of the replacement of the real QD film on top of an SOI planarized waveguide by an effective medium. Indicated are the height difference $l$ between the top surface of the PWG and its silica cladding and the native silica layer in between the PWG top surface and the effective medium representing the QD film. (B) Cross-sectional representation of the simulated electric field for $1520 \mathrm{~nm}$ light guided by a PWG coated by a QD monolayer. Colors represent the field strength, increasing from blue to red. (C) Comparison of the experimental and simulated $\alpha$ spectrum of a QD coated PWG for two different combinations of $l$ and $\varepsilon_{h}$.

As shown in Fig. 3C, a close match can be obtained between the simulated and experimental $\alpha$ spectrum for a QD monolayer-coated PWG by adjusting $\varepsilon_{h}$. It should be noted that the $\varepsilon_{h}$ value needed to match the experimental and simulated $\alpha$ spectrum somewhat depends on the geometry of the PWG. Using AFM, a height 
difference $l$ between the top surface of the PWG and its silica cladding in the range $6-10 \mathrm{~nm}$ has been obtained. Varying $l$ between both values as extreme cases, we obtain agreement between experiment and simulation for $\varepsilon_{h}=1.0(l=6 \mathrm{~nm})$ to $\varepsilon_{h}=1.16(l=10 \mathrm{~nm})$. For QDs capped by oleic acid $(\varepsilon=2.1$ at $2000 \mathrm{~nm})$ [10], both figures are relatively low yet the same holds for the $\varepsilon_{h}=1.5$ found for $\mathrm{PbS}$ QD monolayers deposited on glass (see Fig. 1).

\section{CONCLUSION}

We have studied light absorption in close packed $\mathrm{PbS}$ and $\mathrm{PbS} / \mathrm{CdS} \mathrm{QD}$ monolayers formed by LangmuirBlodgett deposition. The measured absorption enhancement has been explained by dipolar coupling between neighbouring QDs, implying that each QD is driven by a combination of the external optical field and the polarization fields of surrounding QDs. Using similar Langmuir-Blodgett QD monolayers as a top coating on SOI planarized waveguides, we clearly retrieve the QD absorbance in the waveguide losses. The experimental absorption coefficients due to the QD top layer can be simulated using an approach where the QD layer is replaced by an effective medium with an effective dielectric function determined again by dipolar coupling between neighboring QDs. This approach leaves the host dielectric constant $\varepsilon_{h}$ as the only adjustable parameter and provides a generic scheme to model optical properties of composite materials containing close packed QD films.

\section{ACKNOWLEDGEMENTS}

The authors acknowledge EU-FP7 (project Navolchi) and Belgian Science Policy (BelSPo) for funding this research. AO acknowledges the IWT-Vlaanderen for a scholarship. PG acknowledges Ghent University for a scholarship.

\section{REFERENCES}

[1] D.V. Talapin, J.-S. Lee, M.V. Kovalenko, E.V. Shevchenko: Prospects of colloidal nanocrystals for electronic and optoelectronic applications, Chemical Reviews 2009, 110, 389-458.

[2] I. Moreels, Y. Justo, B. De Geyter, K. Haustraete, J.C. Martins, Z. Hens: Size-Tunable, bright, and stable PbS Quantum dots: A surface chemistry study, Acs Nano 2011, 5, 2004-2012.

[3] J.M. Pietryga, D.J. Werder, D.J. Williams, J.L. Casson, R.D. Schaller, V.I. Klimov, J.A. Hollingworth: Utilizing the lability of lead selenide to produce heterostructured nanocrystals with bright, stable infrared emission, Journal of the American Chemical Society 2008, 130, 4879-4885.

[4] Y. Justo, I. Moreels, K. Lambert, Z. Hens: Langmuir-Blodgett monolayers of colloidal lead chalcogenide quantum dots: Morphology and photoluminescence, Nanotechnology 2010, 21, 295606.

[5] K. Lambert, I. Moreels, D. Van Thourhout, Z. Hens: Quantum dot micropatterning on Si, Langmuir 2008, 24, 5961-5966.

[6] I. Moreels, K. Lambert, D. Smeets, D. De Muynck, T. Nollet, J.C. Martins, F. Vanhaecke, A. Vantomme, C. Delerue, G. Allan, Z. Hens: Size-dependent optical properties of colloidal PbS quantum dots, Acs Nano 2009, 3, 3023-3030.

[7] P. Geiregat, Y. Justo, S. Abe, S. Flamee, Z. Hens: Giant and broad-band absorption enhancement in colloidal quantum dot monolayers through dipolar coupling, Acs Nano 2013.

[8] I. Moreels, G. Allan, B. De Geyter, L. Wirtz, C. Delerue, Z. Hens: Dielectric function of colloidal lead chalcogenide quantum dots obtained by a Kramers-Kronig analysis of the absorbance spectrum, Physical Review B 2010, 81, 235319.

[9] B. De Geyter, Z. Hens: The absorption coefficient of PbSe/CdSe core/shell colloidal quantum dots, Applied Physics Letters 2010, 97, 161908.

[10] R. Signorell, A.K. Bertram: Physical chemistry of aerosols, Physical Chemistry Chemical Physics 2009, 11, 7759-7759. 